\title{
ORTHOGONAL EXPANSIONS OF VECTORS IN A HILBERT SPACE FOR NON-GAUSSIAN MEASURES
}

\author{
YOSHIAKI OKAZAKI
}

\begin{abstract}
Let $\mathcal{H}$ be a separable Hilbert space and $\mu$ a probability Radon measure on $\mathcal{K}$ of second order. Then there exist $\left(a_{n}\right) \in l^{2}$, an O.N.S. $\left(x_{n}\right) \subset \mathcal{H}$ and an O.N.S. $\left(\xi_{n}\right) \subset H$ such that the orthogonal series $\sum_{n=1}^{\infty} a_{n} \xi_{n}(x) x_{n}$ converges in $\mathcal{K}$ $\mu$-almost everywhere and it holds that $x=\sum_{n=1}^{\infty} a_{n} \xi_{n}(x) x_{n}, \mu$-almost everywhere, where $H$ is the generating Hilbert space of $\mu$. In the case where $\mu$ is a Gaussian measure, a similar result was proved by Kuelbs [2] in general Banach spaces.
\end{abstract}

1. Let $\mathcal{H}$ be a separable (real or complex) Hilbert space and $\mu$ a probability measure on $\mathcal{H}$ of second order, that is, $\int_{\mathscr{C}}\|x\|^{2} d \mu(x)<+\infty$. $\mathcal{H}^{\prime}$ denotes the dual space of $\mathcal{H}$ and is identified with $\mathcal{H}$ by the conjugate linear isometry $\mathscr{H} \ni x \rightarrow$ $(\mid x) \in \mathcal{H C}^{\prime}$, where $(\mid)$ is the inner product of $\mathcal{H C}$ (Riesz's theorem).

Let $R: \mathcal{H C}^{\prime} \rightarrow L^{2}(\mu)$ be $R(y)=(\mid y)$. We let $H$ be the $L^{2}(\mu)$-closure of $R \mathcal{F C}^{\prime}$. Then $R: \mathcal{F C}^{\prime} \rightarrow H$ is a continuous (conjugate) linear mapping since

$$
\|R(y)\|^{2}=\int|(x \mid y)|^{2} d \mu(x) \leqslant\left(\int\|x\|^{2} d \mu(x)\right)\|y\|^{2} .
$$

THEOREM. There exist $\left(a_{n}\right) \in l^{2}$, an O.N.S. $\left(x_{n}\right) \subset \mathcal{H}$ and an O.N.S. $\left(\xi_{n}\right) \subset H$ such that

$$
x=\sum_{n=1}^{\infty} a_{n} \xi_{n}(x) x_{n}, \quad \mu \text {-almost everywhere, }
$$

where the right-hand side converges in $\mathcal{H}$ for $\mu$-almost every $x$.

Proof. First we show that $R: \mathcal{H}^{\prime} \rightarrow H$ is a Hilbert-Schmidt operator. Let $\left(e_{n}\right)$ be a C.O.N.S. in $\mathcal{H}^{\prime}$, then we have

$$
\sum_{n=1}^{\infty}\left\|R\left(e_{n}\right)\right\|^{2}=\sum_{n=1}^{\infty} \int\left|\left(x \mid e_{n}\right)\right|^{2} d \mu(x)=\int\|x\|^{2} d \mu(x)<+\infty,
$$

which proves the assertion.

By the well-known representation theorem of a Hilbert-Schmidt operator, there exist $\left(a_{n}\right) \in l^{2}$, an O.N.S. $\left(x_{n}\right) \subset \mathcal{K}$ and an O.N.S. $\left(\xi_{n}\right) \subset H$ such that

$$
R(y)(x)=\sum_{n=1}^{\infty} a_{n}\left(x_{n} \mid y\right) \xi_{n}(x) \quad \text { in } H \subset L^{2}(\mu) \text {. }
$$

Received by the editors December 1, 1981 and, in revised form, March 5, 1982.

1980 Mathematics Subject Classification. Primary 60B11.

Key words and phrases. Orthogonal expansion, generating Hilbert space.

(C)1982 American Mathematical Society $0002-9939 / 82 / 0000-0554 / \$ 01.75$ 
We prove that the orthogonal series $\sum_{n=1}^{\infty} a_{n} \xi_{n}(x) x_{n}$ converges in $\mathcal{C} \mu$-almost everywhere. Since

$$
\left\|\sum_{n=k}^{m} a_{n} \xi_{n}(x) x_{n}\right\|^{2}=\sum_{n=k}^{m} a_{n}^{2}\left|\xi_{n}(x)\right|^{2},
$$

it is sufficient to show the series $\Sigma a_{n}^{2}\left|\xi_{n}(x)\right|^{2}$ converges $\mu$-almost everywhere. By the equality

$$
\int \sum_{n=k}^{m} a_{n}^{2}\left|\xi_{n}(x)\right|^{2} d \mu(x)=\sum_{n=k}^{m} a_{n}^{2}
$$

and by $\left(a_{n}\right) \in l^{2}$, it follows that the sequence $\sum_{n=1}^{k} a_{n}^{2}\left|\xi_{n}(x)\right|^{2}, k=1,2, \ldots$, converges in $L^{1}(\mu)$. Hence there is a subsequence which converges $\mu$-almost everywhere. But the sequence is nonnegative and nondecreasing, so the sequence itself converges $\mu$-almost everywhere.

Lastly we show that $x=\sum_{n=1}^{\infty} a_{n} \xi_{n}(x) x_{n}, \mu$-almost everywhere. Let $\left(e_{k}\right)$ be a C.O.N.S. in $\mathcal{H}^{\prime}$. Then we have for $\mu$-almost every $x$,

$$
\begin{aligned}
\left(x \mid e_{k}\right) & =R\left(e_{k}\right)(x)=\sum_{n=1}^{\infty} a_{n}\left(x_{n} \mid e_{k}\right) \xi_{n}(x) \\
& =\left(\sum_{n=1}^{\infty} a_{n} \xi_{n}(x) x_{n} \mid e_{k}\right), \quad k=1,2, \ldots
\end{aligned}
$$

Since $\left(e_{k}\right)$ separates $\mathcal{H}$, we get the assertion.

This completes the proof.

2. The Hilbert space $H$ in the preceding section coincides with the generating Hilbert space of $\mu$ constructed by Kuelbs [3, Lemma 2.1]. So by the representation of the mapping $R$ obtained by Kuelbs [3], we can determine $\left(a_{n}\right),\left(x_{n}\right)$ and $\left(\xi_{n}\right)$ explicitly as follows. Let $R^{*}: H^{\prime} \rightarrow \mathcal{H}$ be the adjoint linear operator and set $T=$ $R \circ R^{*}: H^{\prime}=H \rightarrow \mathscr{H}=\mathcal{H}^{\prime} \rightarrow H$. Then it holds that

$$
R^{*}(\xi)=\int x \xi(x) d \mu(x)
$$

(Bochner integral, Kuelbs [3, (2.1)]),

$$
T(\xi)(x)=\int(x \mid y) \overline{\xi(y)} d \mu(y), \quad \xi \in H
$$

(Kuelbs [3, (2.2)]).

Then $T: H \rightarrow H$ (or $\left.L^{2}(\mu) \rightarrow L^{2}(\mu)\right)$ is a positive selfadjoint nuclear linear operator (in fact, $T$ is an integral operator with the integrable kernel $(x \mid y), x, y \in \mathcal{H}_{C}$ ). Let $c_{n} \geqslant 0, \sum_{n=1}^{\infty} c_{n}<+\infty$ be the eigenvalues and $\left(\xi_{n}\right) \subset H$ the eigenfunctions of $T$. Put $x_{n}=c_{n}^{-1 / 2} R^{*}\left(\xi_{n}\right)$. Then we have

$$
\begin{aligned}
\left(x_{n} \mid x_{m}\right) & =c_{n}^{-1 / 2} c_{m}^{-1 / 2}\left(\int x \xi_{n}(x) d \mu(x) \mid \int y \xi_{m}(y) d \mu(y)\right) \\
& =c_{n}^{-1 / 2} c_{m}^{-1 / 2} \int\left[\int(x \mid y) \overline{\xi_{m}(y)} d \mu(y)\right] \xi_{n}(x) d \mu(x) \\
& =c_{n}^{-1 / 2} c_{m}^{-1 / 2} c_{m} \int \overline{\xi_{m}(x)} \xi_{n}(x) d \mu(x)=\delta_{n, m} .
\end{aligned}
$$


So we get $x=\Sigma c_{n}^{-1 / 2} \xi_{n}(x) x_{n} \mu$-almost everywhere. Note that this procedure is very similar to Kuelbs [2, §4].

3. Let $\nu$ be the Gaussian Radon measure on $\mathcal{H}$ with the characteristic functional $\exp \left(-\int\left|\left\langle x, x^{\prime}\right\rangle\right|^{2} d \mu(x) / 2\right), x^{\prime} \in \mathcal{H}^{\prime}$. Then by Kuelbs [2, Theorem 3.1], it follows that $x=\sum a_{n} \xi_{n}(x) x_{n} \nu$-almost everywhere. Kuelbs' arguments are as follows. For the Gaussian measure $\nu$, the orthogonality of $\left(\xi_{n}\right)$ in $L^{2}(\nu)$ is equivalent to the independence of $\left(\xi_{n}\right)$ as random variables on $(\mathcal{H}, \nu)$. And by the convergence theorem of sums of independent random variables due to Ito and Nisio [1], it follows that $x=\sum a_{n} \xi_{n}(x) x_{n} \nu$-almost everywhere. The purpose of this note is to show $x=\sum a_{n} \xi_{n}(x) x_{n}$ for $\mu$-almost every $x$. Note that $\nu(A)=1$ does not imply $\mu(A)=1$ in general. Note also that the orthogonal random variables $\left(\xi_{n}\right)$ are not independent over $(\mathcal{H}, \mu)$, so we cannot use Ito and Nisio [1]. To prove our theorem the Hilbert-Schmidtness of the operator $R$ is essential. Our result does not hold for general Banach space $\mathcal{H}$.

\section{REFERENCES}

1. K. Ito and M. Nisio, On the convergence of sums of independent Banach space valued random variables, Osaka J. Math. 5 (1968), 35-48.

2. J. Kuelbs, Expansions of vectors in a Banach space related to Gaussian measures, Proc. Amer. Math. Soc. 27 (1971), 364-371.

3. _ A strong convergence theorem for Banach space valued random variables, Ann. Probab. 4 (1976), 744-771.

Department of Mathematics, Kyushu University 33, FukUoka 812, JaPan 